\title{
ON A THEOREM OF PLESSNER*
}

\section{BY NELSON DUNFORD}

If $f(t)$ is a real-valued function of a real variable, periodic with period $2 \pi$ and of bounded variation, then $f(t)$ is absolutely continuous provided $v(u)$, the total variation of $f(t+u)-f(t)$ on any interval of length $2 \pi$, tends to zero with $u$. This theorem, which is the converse of a well known theorem of Lebesgue, has been proved by Plessner, and by Wiener and Young. $\dagger$ Ursell $\ddagger$ has given some interesting results concerning the total variation of $f(t+u)-f(t)$ for measurable functions $f(t)$, which when combined with the above theorem show (as he has pointed out) that that theorem holds for measurable functions. The papers referred to contain the essential ideas sufficient for a very short proof of the general theorem. In fact, with two additional lemmas which are given below, the proof as given by Plessner holds in the general case.

By an admissible function will be meant one which is finitevalued and periodic with period $2 \pi$.

TheOREM 1. If $f(t)$ is admissible and $\lim _{u=0} v(u)=0$, then $v(u)$ is continuous.

Let $u_{1}, u_{2}$ be any real numbers and $\delta_{i}=\left(t_{i-1}, t_{i}\right)$ be a partition of the interval $(-\pi, \pi)$. Then, if $t_{i}^{\prime}=t_{i}+u_{1}$, the intervals $\delta_{i}^{\prime}=\left(t_{i-1}^{\prime}, t_{i}^{\prime}\right)$ form a partition of $\left(-\pi+u_{1}, \pi+u_{1}\right)$ and $\S$

$$
\begin{aligned}
\delta_{i}\left\{f\left(t+u_{1}+u_{2}\right)-f(t)\right\}= & \delta_{i}\left\{f\left(t+u_{1}\right)-f(t)\right\} \\
& +\delta_{i}^{\prime}\left\{f\left(t+u_{2}\right)-f(t)\right\},
\end{aligned}
$$

and so $v\left(u_{1}+u_{2}\right) \leqq v\left(u_{1}\right)+v\left(u_{2}\right)$. This shows that $v$ is finite everywhere; for if $v(u)<K$ on $(-a, a)$, then $v(u)<2 K$ on $(-2 a, 2 a)$.

* Presented to the Society, April 20, 1935.

$\dagger$ Plessner, Eine Kennzeichnung der totalstetigen Funktionen, Journal für Mathematik, vol. 160 (1929), pp. 26-32. Wiener and Young, The total variation of $g(x+h)-g(x)$, Transactions of this Society, vol. 35 (1933), pp. 327-340.

$\ddagger$ Ursell, On the total variation of $f(t+\tau)-f(t)$, Proceedings of the London Mathematical Society, (2), vol. 37 (1934), pp. 402-415.

$\S$ If $\delta=(a, b)$, by $\delta f(t+u)$ is meant $f(b+u)-f(a+u)$. 
From the above inequality it follows that $\left|v(u)-v\left(u_{1}\right)\right|$ $\leqq v\left(u-u_{1}\right)+v\left(u_{1}-u\right)$, which shows that $v(u)$ is continuous.

LEMмa 1. If the function $f(t)$ is admissible and measurable and $\lim _{u=0} v(u)=0$, then $f(t)$ is uniformly continuous.

If not, there exists a positive number $\gamma$ such that for every $\alpha>0$ there is an interval $\delta=\left(t, t^{\prime}\right)$ in $(-\pi, \pi)$ with $\left|t-t^{\prime}\right|<\alpha$ and such that $|\delta f(t)|>\gamma$. There is an $\alpha_{1}>0$ such that $v(u)<\gamma / 3$ if $|u| \leqq \alpha_{1}$. Let $\beta$ be a positive number $<\alpha_{1} / 2$ and $<\gamma / 2$. Then by the theorem of Lusin $\left|f(t)-f\left(t^{\prime}\right)\right|<\beta$ for all $t, t^{\prime}$ in the interval $I=(-2 \pi, 2 \pi)$ except those in a set $E_{\beta}$ with measure less than $\beta$, provided $\left|t-t^{\prime}\right|$ is sufficiently small, say $<\alpha_{2}$. Fix an interval $\delta=\left(t, t^{\prime}\right)$ such that $\left|t-t^{\prime}\right|<\alpha_{2}$ and $|\delta f(t)|>\gamma$. Then with this $\delta$ and any $u$ such that $|u| \leqq \alpha_{1}$, we have

$$
\gamma / 3>v(u) \geqq|\delta\{f(t+u)-f(t)\}|>\gamma-|\delta f(t+u)| .
$$

If there exists a $u$ with $|u| \leqq \alpha_{1}$ such that $t+u, t^{\prime}+u$ are both in $I-E_{\beta}$, then the above inequality gives the contradiction $\gamma / 3>\gamma-\beta>\gamma / 2$. To see that such a $u$ exists define a one-toone correspondence between the intervals $\Delta=\left(t-\alpha_{1}, t+\alpha_{1}\right)$ and $\Delta^{\prime}=\left(t^{\prime}-\alpha_{1}, t^{\prime}+\alpha_{1}\right)$ by the equations $x=t+u, x^{\prime}=t^{\prime}+u$. If $E$ is a set in $\Delta, E^{\prime}$ will denote the corresponding set in $\Delta^{\prime}$. Now if no such $u$ exists $E_{\beta} \supset E_{\beta} \Delta+\left(\Delta-E_{\beta} \Delta\right)^{\prime}$ and so

$$
\begin{aligned}
\alpha_{1} / 2>\beta>m\left(E_{\beta}\right) & \geqq m\left[E_{\beta} \Delta+\left(\Delta-E_{\beta} \Delta\right)^{\prime}\right] \\
& \geqq\left[m\left(E_{\beta} \Delta\right)+m\left(\Delta-E_{\beta} \Delta\right)^{\prime}\right] / 2=\alpha_{1},
\end{aligned}
$$

which again is a contradiction.

THEOREM 2. If $f(t)$ is admissible and measurable and if also $\lim _{u=0} v(u)=0$, then $f(t)$ is absolutely continuous.

The proof is entirely analogous to that of Plessner. By Lemma $1, f(t)$ is summable and if $s_{n}^{\prime}(t)$ is the first arithmetic mean of its Fourier series, then

$$
s_{n}^{\prime}(t)-f(t)=\int_{-\pi}^{+\pi}\{f(t+u)-f(t)\} F_{n}(u) d u,
$$

where $F_{n}(u)$ is Fejér's kernel; and thus the total variation 


$$
T\left[s_{n}^{\prime}(t)-f(t)\right] \leqq \int_{-\pi}^{+\pi} v(u) F_{n}(u) d u \rightarrow 0
$$

by Fejér's theorem, since $v(u)$ is continuous and $v(0)=0$. For $\epsilon>0$ there is an $n_{0}$ and a $\delta>0$ such that

$$
T\left[s_{n_{0}}^{\prime}(t)-f(t)\right]<\epsilon / 2, \quad \sum\left|\delta_{i} s_{n_{0}}{ }^{\prime}(t)\right|<\epsilon / 2
$$

for an arbitrary set $\left\{\delta_{i}\right\}$ of non-overlapping intervals with $\sum\left|\delta_{i}\right|<\delta$. Thus, for such a set of intervals, ${ }^{*}$

$$
\sum\left|\delta_{i} f(t)\right| \leqq \sum\left|\delta_{i}\left\{f(t)-s_{n_{0}^{\prime}}(t)\right\}\right|+\sum\left|\delta_{i} s_{n_{0}}{ }^{\prime}(t)\right|<\epsilon .
$$

It might be pointed out that the theorem as stated is no less general than the corresponding theorem where $f(t)$ is finite and measurable on $(a, b)$ and $v(u)$ is the total variation of $f(t+u)$ $-f(t)$ on $(a, b-u)$.

The following example which is similar to one given by Ursell is interesting in connection with Theorem 2. Let $\left\{x_{a}\right\}$ be a Hamel $\dagger$ base with $x_{1}=\pi$, so that every real number is expressible uniquely as a finite linear combination of elements of $\left\{x_{a}\right\}$ with rational coefficients. If $t=r_{1} \pi+\sum r_{i} x_{i}$ and $f(t)=r_{1}-t / \pi$, then $f(t)$ is admissible and additive, that is, $f(t+u)=f(t)+f(u)$, and hence $v(u)$ is identically zero. Theorem 2 shows that $f(t)$ is not measurable and the example shows that the hypothesis of measurability is essential in Theorem 2 as well as in Lemma 1.

BROWN UNIVERSITY

* My attention is called, by Hans Lewy, to the fact that the use of Fourier series may be avoided by approximating to $f(t)$ by means of the absolutely continuous functions $(1 / h) \int_{0}^{h} f(t+u) d u$.

$\dagger$ Hamel, Basis aller Zahlen und der unstetigen Lösungen der funktional Gleichungen $F(x+y)=F(x)+F(y)$, Mathematische Annalen, vol. 60 (1905), p. 459. 